East African Medical Journal Vol. 86 No. 10 October 2009

PREGNANCY OUTCOMES IN MOTHERS WITH ADVANCED HUMAN IMMUNODEFICIENCY VIRUS DISEASE

J.W. Musana, MBChB, MMed, (UoN), Senior Registrar, S.B.O. Ojwang, MD, MMed, (UoN), Dip. Gyn. Oncol. (Sweden), Professor, W. Khisa, MBChB, MMed, (UoN), Lecturer, Department of Obstetrics and Gynaecology, College of Health Sciences, University of Nairobi, P. O. Box 19676 - 00202, Nairobi, Kenya and J.N. Kiarie, MBChB, (UoN), Consultant and Head of Department, Obstetrics and Gynaecology, Kenyatta National Hospital, P. O. Box 20723-00202, Nairobi, Kenya

Request for reprints to: Dr. J.W. Musana, Department of Obstetrics and Gynaecology, College of Health Sciences, University of Nairobi, P. O. Box 19676 - 00202, Nairobi, Kenya

\title{
PREGNANCY OUTCOMES IN MOTHERS WITH ADVANCED HUMAN IMMUNODEFICIENCY VIRUS DISEASE
}

\author{
J.W. MUSANA, S.B.O. OJWANG, W. KHISA and J.N. KIARIE
}

\begin{abstract}
Objective: To determine the impact of HIV disease on immediate maternal and foetal outcomes at the Kenyatta National Hospital, Nairobi, Kenya

Design: Prospective cohort study.

Setting: Kenyatta National Hospital, Nairobi, Kenya, between September 2004 and April 2005.

Subjects: Sixty eight mothers with advanced HIV disease (WHO clinical stage 3 and 4) and 68 HIV negative pregnant mothers.

Results: Mothers with advanced HIV disease were more likely to be anaemic (55\% vs. $16 \% \mathrm{p}<0.001)$, to have sexually transmitted diseases $(56 \%$ vs. $15 \%, \mathrm{p}=0.004)$, to have chorioamnionitis $(14.8 \%$ vs. $2 \%, p=0.004)$, to develop preterm premature rupture of membranes ( $31 \%$ vs. $9 \%, \mathrm{p}<0.001)$, to have puerperal pyrexia $(16 \%$ vs. $2 \%, \mathrm{p}=0.032)$ an to die $(5 \%$ vs. $0.5 \%, \mathrm{p}=0.028)$ compared to HIV negative mothers. The mean gestational age at deliver was lower in mothers with advanced HIV disease compared to the seronegative counterparts ( $73 \%$ vs. $32 \%$, delivery $<37$ weeks, $p<0.001)$. Infants of mothers with advanced HIV disease compared to infants of seronegative mothers were more likely to be low birth weight infants $(58 \%$ vs. $21 \%, \mathrm{p}<0.001)$, stillborn ( $4 \%$ vs. $2 \%, \mathrm{p}=0.308)$ and to have low Apgar scores $(28 \%$ vs. $12 \%$, Apgar score $<4$ at 5 minutes $\mathrm{p}=0.02)$. Perinatal sepsis and perinatal deaths were more common in infants born to mothers with advanced HIV disease compared to infants born to HIV negative mothers ( 8 vs. $3, p=0.003$ and 14 vs. $5, p=0.025$ respectively). External congenital anomalies were similar in the two groups $(5.9 \%$ vs. $5.9 \%)$.

Conclusion: Pregnancies complicated by advanced HIV disease are more likely to have adverse outcomes, both maternal and foetal. Advanced HIV disease is associated with increased risk of both maternal and fetal mortality. HIV infected mothers should be counselled on the increased pregnancy risks associated with advanced disease.
\end{abstract}

\section{INTRODUCTION}

Pregnancy outcomes in mothers with HIV infection have been a subject of many studies both in the developed and the developing world. Differences exist in results in studies on pregnancy outcomes in HIV infected women from Africa compared to those from Europe and the USA(1). Whereas most studies in Africa have shown increased adverse outcomes, most Western studies have shown little or no demonstrable impact of HIV infection on pregnancy and on maternal and neonatal outcomes (1).

Neonatal morbidity such as prematurity, low birth weight, low Apgar scores, stillbirths, neonatal sepsis, perinatal and neonatal mortality and congenital anomalies have been the focus of the comparison studies between HIV seropositive and seronegative mothers. Maternal morbidity including anaemia, premature rupture of membranes, sexually transmitted disease, chorioamnionitis, puerperal pyrexia, postpartum haemorrhage, length of hospital stay and maternal mortality have also been studied extensively. All these factors have a significant bearing on child survival and maternal well-being. The Western studies which have focused on pregnant mothers with HIV disease are small in number and have included small number of pregnant mothers. They may hence lack statistical power to detect a true difference both in foetal and maternal outcome characteristics (1). Most African studies reported 
increased adverse outcomes. However, a few have shown no significant difference in pregnancy outcome HIV infected mothers and their healthy HIV negative controls. None of these studies focused, exclusively on mothers with advanced HIV disease where adverse impact is likely to be maximal.

This study focused on pregnant mothers with advanced HIV disease (WHO clinical stage 3 and 4). The objective of the study was to determine the impact of advanced HIV disease on immediate maternal and foetal outcomes. It was a prospective cohort study conducted at the Kenyatta National Hospital from September 2004 to April 2005.

\section{MATERIALS AND METHODS}

Mothers with advanced HIV disease were recruited from the antenatal wards of Kenyatta National Hospital by the principal investigator assisted by two nurses. Information regarding the research was given to the participants and those that agreed to participate signed on informed consent form.

Once a mother with advanced HIV disease was identified, a control matched for age \pm 5 years and gestation by dates \pm 4 weeks was identified and interviewed from the antenatal clinic. The first part of the structured questionnaire was then administered. Mothers with advanced HIV disease and those who were HIV negative were followed up by the principal investigator and the nurses until delivery or until the final outcome of the pregnancy was known. The second part of the structured questionnaire was filled and completed one week after delivery when the final status of the mother and the infant was determined.

The raw data from the questionnaires were then verified, coded and entered into the computer using Epi Info version 6 and further analysis was done using the SPSS programme. Two by two tables were used to test for associations and odds ratio calculations were performed. Chi-squared test was used for proportional variables and the t-test for continuous variables. A P-value of 0.05 or less was taken to be significant.

\section{RESULTS}

Mothers who met the study criteria were interviewed. A total of 177 mothers were invited to participate but 17 mothers declined to participate citing personal reasons and lack of direct benefit of the study to them. The women who agreed to participate were followed up over a period of 32 weeks.
Twenty three mothers were lost to follow up. One hundred and thirty eight mothers were seen during delivery and 137 mothers one week after delivery. One HIV negative mother absconded before the end of the follow up period while the delivery notes of one infant born to a HIV negative mother got lost. Analysis was hence done on a total of 136 mothers, 68 of them HIV negative and 68 with advanced HIV disease.

Table 1 shows the sociodemographic and antenatal characteristics of the study participants. Mothers with advanced HIV disease were more likely to be older than the HIV negative mothers ( 27 years vs. 24 years $p=0.009$ ). This difference was significant. There was no difference in the marital status between the two groups. HIV negative mothers were more educated than mothers with advanced HIV disease and this difference was significant $(\mathrm{p}=0.004)$. Primigravidas were more among the HIV negative mothers compared to mothers with advanced HIV disease $(p=0.001)$. Mothers with advanced HIV disease were more likely to have had previous abortions compared to the HIV negative mothers and this difference achieved statistical significance $(\mathrm{p}=0.008)$. There was no difference in the VDRLstatus in the two groups but the incidence of candidiasis and trichomoniasis was higher in mothers with advanced HIV disease ( $41 \%$ vs. $12 \%, \mathrm{p}<0.001, \mathrm{RR}=3.5$ ). Mothers with advanced HIV disease were more likely to be anaemic compared to the HIV negative mothers ( $55 \%$ vs. $16 \%, \mathrm{p}<0.001)$. The study participants were enrolled at more or less the same gestation.

More seronegativemothersunderwentinduction of labour compared to mothers with advanced HIV disease (16\% vs. $13 \%, \mathrm{p}=0.6)$. In total there were more Caesarean sections done in the seronegative group compared to the advanced HIV group (41\% vs. $13 \%$ $p<0.001, R R=0.395 \%$ CI0.2-0.6). No operative vaginal delivery was performed in the advanced HIV group while there were three vacuum deliveries in the HIV negative group.

Mothers with advanced HIV disease were more likely to have preterm premature rupture of membranes compared to the HIV negative mothers ( $10 \%$ vs. $6 \%$ p=0.5). Women with advanced HIV disease were more prone to preterm premature rupture of membranes and this difference achieved statistical significance ( $31 \%$ vs. $10 \% \mathrm{p}=0.003, \mathrm{RR} 3$, 95\% CI1.4-6.6). Chorioamnionitis was found to occur more in mothers with advanced HIV disease $(10 \%$ vs. $6 \% \mathrm{p}=0.5)$ 


\section{Figure 1}

Enrollment and follow-up of participants

Invited to participate

Agreed to participate

Seen during delivery

Seen one week after delivery

Lost to follow-up
Advanced HIV

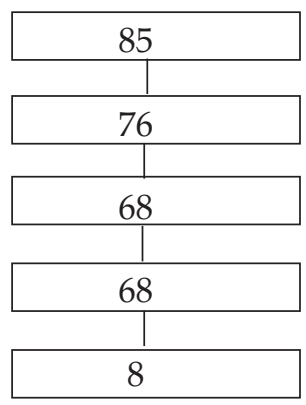

HIV negative

Table 1

Study population characteristics

\begin{tabular}{lcccccc}
\hline \multirow{2}{*}{ Characteristic } & \multicolumn{2}{c}{ Women with advanced } & \multicolumn{2}{c}{ HIV negative } & P-value & Relative \\
& HIV disease $(\mathrm{n}=68)$ & women $(\mathrm{n}=68)$ & & risk/95\% CI \\
& No. & $(\%)$ & No. & $(\%)$ & & \\
\hline Above primary education & 6 & 9 & 19 & 28 & 0.004 & $0.3(0.1-0.7)$ \\
Married & 53 & 78 & 52 & 78 & 1 & $1(0.8-1.2)$ \\
Unemployed & 44 & 65 & 42 & 63 & 0.8 & $1(0.8-1.3)$ \\
Primigravida & 9 & 14 & 34 & 50 & $<0.001$ & $0.3(0.1-0.5)$ \\
Had previous abortion & 13 & 22 & 4 & 6 & 0.008 & $3.7(1.3-10.7)$ \\
Haemoglobin $<10 \mathrm{~d} / \mathrm{dl}$ & 37 & 55 & 11 & 16 & $<0.001$ & $3.4(1.9-6.0)$ \\
VDRL positive & 2 & 3 & 1 & 1 & 0.0308 & $0.8(1.2-1.7)$ \\
Trichomoniasis or & 28 & 41 & 8 & 12 & $<0.001$ & $3.5(1.7-7.1)$ \\
candidiasis & & & & & & \\
Mean age (years) & 27.03 & & 24.41 & & 0.009 & $0.9(0.8-1)$ \\
Mean gestation at & 31.1 & & 31.2 & & 0.811 & $1.0(0.9-1.1)$ \\
enrollment (weeks) & &
\end{tabular}

Table 2

Maternal pregnancy outcomes

\begin{tabular}{lcccccc}
\hline Characteristic & \multicolumn{2}{c}{$\begin{array}{c}\text { Advanced HIV } \\
(\mathrm{n}=68)\end{array}$} & \multicolumn{2}{c}{$\begin{array}{c}\text { HIV negative } \\
(\mathrm{n}=68)\end{array}$} & P-value & $\begin{array}{c}\text { Relative } \\
\text { risk/95\% CI }\end{array}$ \\
& No. & $(\%)$ & No. & $(\%)$ & & \\
\hline $\begin{array}{l}\text { Postpartum pyrexia } \\
\text { temp }>38^{\circ} \mathrm{C}\end{array}$ & 15 & 23 & 6 & 9 & 0.03 & $2.5(1.0-6.0)$ \\
Hospitalisation $>$ week 1 & 23 & 44 & 11 & 27 & 0.1 & $1.6(0.9-2.9)$ \\
Maternal deaths & 6 & 9 & 0 & 0 & 0.028 & NIL \\
Caesarean section delivery & 9 & 13 & 28 & 41 & $<0.001$ & $0.3(0.2-0.6)$ \\
Labour induction & 7 & 13 & 10 & 16 & 0.6 & $0.8(0.3-1.9)$ \\
Operative vaginal delivery & 0 & 0 & 3 & 4.5 & 0.2 & NIL \\
Preterm PROM & 7 & 10 & 4 & 6 & 0.5 & $17(0.5-5.7)$ \\
Term PROM & 21 & 31 & 7 & 10 & 0.003 & $3(1.4-6.6)$ \\
Chorioamnionitis & 7 & 10 & 4 & 6 & 0.5 & $1.7(0.5-5.7)$ \\
Meconium staining in liquor & 22 & 35 & 14 & 20 & 0.07 & $1.7(0.9-3.0)$ \\
Hypertensive disease & 9 & 14 & 8 & 12 & 0.7 & $1.0(0.9-1.1)$ \\
DBP>90mmHg & & & & & & \\
\hline
\end{tabular}


Table 3

Foetal pregnancy outcomes

\begin{tabular}{|c|c|c|c|c|c|c|}
\hline \multirow[t]{2}{*}{ Characteristic } & \multicolumn{2}{|c|}{$\begin{array}{l}\text { Advanced HIV } \\
(\mathrm{n}=68)\end{array}$} & \multicolumn{2}{|c|}{$\begin{array}{l}\text { HIV negative } \\
\quad(\mathrm{n}=68)\end{array}$} & \multirow[t]{2}{*}{ P-value } & \multirow[t]{2}{*}{$\begin{array}{c}\text { Relative } \\
\text { risk } 95 \% \text { CI }\end{array}$} \\
\hline & No. & $(\%)$ & No. & $(\%)$ & & \\
\hline $\begin{array}{l}\text { Gestation at } \\
\text { delivery }<37 \text { weeks }\end{array}$ & 48 & 73 & 22 & 22 & $<0.001$ & $2.2(1.5-3.3)$ \\
\hline $\begin{array}{l}\text { Low birth weight } \\
<2500 \text { gm }\end{array}$ & 38 & 58 & 14 & 21 & $<0.001$ & $2.8(1.7-4.7)$ \\
\hline $\begin{array}{l}\text { Apgar score }<4 \text { at } 5 \\
\text { minutes }\end{array}$ & 18 & 27 & 8 & 12 & 0.02 & $2.3(1.1-5.0)$ \\
\hline Congenital anomalies & 4 & 6 & 4 & 6 & 1 & NIL \\
\hline Neonatal sepsis & 8 & 12 & 3 & 4 & 0.1 & $2.7(0.7-9.6)$ \\
\hline Neonatal deaths & 13 & 22 & 3 & 6 & 0.03 & $3.5(1.0-11.4)$ \\
\hline
\end{tabular}

Therewasnostatistical differenceinhypertensive disease and hospital stay for more than a week between the two groups. There were six maternal deaths in the advanced HIV group and no maternal deaths in the HIV seronegative group. This difference was statistically significant $(p=0.028)$. Three mothers passed away due to complications of pulmonary tuberculosis, two due to bacterial meningitis and one due to presumptive pneumocystis carinii pneumonia (PPCP).

Mothers with advanced HIV disease tended to deliver earlier compared to HIV negative mothers (73\% vs. $32 \% \mathrm{p}<0.001 \mathrm{RR}=2.2,95 \%$ CI 1.5-3.3). The mean gestation at delivery was 35 weeks for mothers with advanced HIV disease compared to 38 weeks for HIV negative mothers $(p<0.001)$. Infants of mothers with advanced HIV disease compared to infants of HIV negative mothers were more likely to be low birth weight infants $38(56 \%)$ vs. $14(21 \%)$ birth weight $<2500 \mathrm{gm} \mathrm{p}<0.001)$. Infants born to mothers with advanced HIV disease tended to have lower Apgar scores and this was significant for Apgar score less than four at five minutes ( 18 vs. $8, p=0.02)$. There was no difference in the rate of congenital anomalies between the two groups $(5.9 \%$ vs. 5.9\%). Infants of mothers with advanced HIV, disease suffered significant neonatal sepsis ( $12 \%$ vs. $4 \% \mathrm{p}=0.1)$ and early neonatal deaths ( $22 \%$ vs. $6 \% \mathrm{p}=0.03 \mathrm{RR}=3.5$, 95\% CI 1.0-11.4) compared to infants born to mothers who were HIV negative.

\section{DISCUSSION}

This was a prospective cohort study whose aim was to document the immediate impact of advanced HIV disease on pregnancy. Overall, our findings suggest that advanced HIV disease is associated with poor pregnancy outcome both foetal and maternal. We observed that mothers with advanced HIV disease booked with lower haemoglobin levels and this anaemic condition placed then at a higher risk of mortality and morbidity during the antenatal, perinatal and puerperium. Sukwa et al (3) in Ndola Zambia had similar findings. In our study, sexually transmitted infections occurred more significantly in mothers with advanced HIV disease. This is comparable to what Temmermann and colleagues (4) found in the study of maternal HIV infection and pregnancy outcome. Burns et al (5) found that vaginal colonisation with candidiasis occurred frequently in pregnant mothers infected with HIV virus. This collective data strongly suggests that HIV infected pregnant women are at an increased of sexually transmitted disease compared to uninfected women.

Syphilis is more common in HIV positive women in Africa with an incidence of up to $33 \%$ being reported (6). In our study, an increased risk among mothers with advanced HIV disease was found though the difference was not significant. Temmermann and colleagues (4) in Langata, Nairobi, found theincidence of syphilis in HIV positive women to be $3.2 \%$ and also reported that the HIV positive mother was two and a half times more likely to have a positive VDRL test (4). Guay et al (7) in Uganda found that HIV positive mothers were more likely to have been tested for syphilis (22\% vs. $14.5 \%)$. Syphilis is a major cause of perinatal and infant mortality and morbidity and mothers with advanced HIV disease appear to be affected more by the disease.

Term and preterm premature rupture of membranes occurred frequently among mothers with advanced HIV disease. This has adverse implications for mother to child transmission of the virus and foetal survival because of increased incidence of prematurity. Premature rupture of membranes could also have been a contributing factor of increased incidence of chorioamnionitis among mothers with 
advanced disease. Omondi et al (8) also reported that HIV positive mothers tended to have premature rupture of membranes (14.9\% vs. $5 \%$ ).

Our study suggests that advanced HIV disease predisposes to prematurity. More than half of the mothers with advanced HIV disease delivered at less than 37 completed weeks. Thisfinding is in agreement with reports from a larger prospective cohort study conducted in Dar es Salaam, Tanzania (9). In the latter study, prematurity was significantly observed among symptomatic HIV-infected women when compared with HIV -uninfected women. Prematurity is associated with high neonatal mortality and morbidity in our set-up and advanced HIV disease will increase the burden of neonatal care.

In a study by Nyongoet al (10), moderate to severe chorioamnionitis was found in $20 \%$ of preterm and $8 \%$ of term pregnancies. Within preterm deliveries, the placentas showed chorioamnionitis with 37\% of cases where the woman was HIV positive and $18 \%$ of matched seronegative cases. In our study $10 \%$ of mothers with advanced HIV disease had chorioamnionitis compared to $6 \%$ in the HIV negative group. This did not achieve statistical significance $(\mathrm{p}=0.5)$, though it appears that mothers with advanced HIV disease have a higher risk of chorioamnionitis and its associated complications compared to the HIV negative mothers. More mothers with advanced HIV disease suffered post partum pyrexia $\left(\right.$ Temp $\left.>38^{\circ} \mathrm{C}\right)$ compared to their seronegative counterparts $(p=0.03)$. This could possibly be attributed to the higher risk of chorioamnionitis in the same mothers.

Our study recorded six maternal deaths in the advanced HIV group and no maternal deaths in the HIV negative group. This difference was statistically significant $(\mathrm{p}=0.028)$. Thisfinding is in agreement with some Western studies which also recorded higher maternal mortality in pregnant mothers with AIDS $(11,12)$. In our study, three mothers passed away from complications of pulmonary tuberculosis, two from bacterial meningitis and one due to presumptive pneumocystis carinii pneumonia.

Many studiesindeveloped countries have shown little difference in birth weights of babies born to HIV positive mothers compared to infants born to uninfected mothers. However, Minkoff et al (13) found high rates of low birth weight in mothers who were HIV infected. Low birth weight is mostly reported in sub-Saharan African countries and especially among symptomatic mothers or mothers with AIDS. In this study, the difference in birth weight was significant, with mothers with advanced HIV disease giving birth to more low birth weight babies compared to HIV uninfected mothers. These findings are similar to those of Temmermann et al (14) in Nairobi, Guay et al (7) in Uganda, Chamiso (15) in Ethiopia, Jenny et al (9) in Tanzania and Taha (16) in Malawi. However, Lepage (2) in Rwanda found difference in birth weights between HIV positive and HIV negative mothers.

The five minuteApgar score was poorer in infants born to mothers with advanced HIV disease compared to infants of HIV uninfected mothers. Similar findings were reported by Guayet al (7) in Uganda and Chamiso (15) in Ethiopia, however Temmermann et al (17) did not find any significant difference in Apgar scores between infants of seropositive and seronegative mothers.

Though there were no significant differences in occurrence of congenial anomalies between the two groups, neonatal sepsis and early neonatal deaths occurred frequently in infants born to mothers with advanced HIV disease. It is possible that preterm delivery, chorioamnionitis with amniotic fluid infection could account for higher rates of neonatal deaths and neonatal sepsis seen in infants born to mothers with advanced HIV disease. This association was recently reported in one large controlled study, where pregnancies from 457 seropositive women were compared with those of 615 seronegative women matched for age and parity. Not only was chorioamnionitis more common in the former group, but also babies, were more frequently premature and the neonatal death rate was increased (18).

A higher proportion of HIV infected pregnant women in sub-Saharan Africa are likely to consist of those who are in an advanced clinical and immunological stage of the disease and many of them enter pregnancy when they are immunosuppressed. This is compounded by a higher prevalence of malnutrition, opportunistic infections and poor access to treatment. These factors in various degrees of combination have adverse impact on pregnancy outcome regardless of the mothers' HIV status.

Overall, our data show that pregnancies complicated by advanced HIV disease have significant increased risk of maternal and foetal mortality and morbidity. The biological mechanisms of HIV as a cause of adverse pregnancy outcome are not yet fully understood but it is clear from many studies that statistical detectable biological effects upon pregnancy occurs in mothers with HIV infection and more so, those who are in advanced stage of the disease. Larger on-going natural history studies of HIV infected cohorts of pregnant women, such as the National Institutes of Health (NIH) sponsored Women and Infants Transmission Study are hoped to provide more definitive answers regarding the relationship between HIV infection and pregnancy (19). 


\section{REFERENCES}

1. Margaret, A. Johnson and Frank, D. Johnstone. HIV infection in women, First edition, Churchill Livingstone Edinburgh London, 1993; 14:188-198.

2. Lepage, P., Dabis, F., Hitimana, D.G., et al. Perinatal transmission of HIV -1: Lack of impact on maternal HIV infection on characteristics of live births and on neonatal mortality in Kigali, Rwanda. AIDS. 1991; 5: 295-300.

3. Sukwa, T.Y., Baketty, L., Kanyama, I. and Smdal, H. H. Maternal HIV infection and pregnancy outcome. Central. Afr. J. Med. 1996; 48: 233-235.

4. Temmermann, M., Lopital, M.I., Sanghvi, H.C.G., et al. The role of maternal, syphilis, gonorrhea and HIV-1 infection in spontaneous abortion. Int. J. STD. AIDS. 1999; 3: 418-422.

5. Burns, D.N., Toumala R., Chang, B.H., et al. Vaginal colonization or infection with Candida Albicans in HIV infected women during pregnancy and postpartum period. Women and Infant Transmission Study Group. Clin. Infect. Dis. 1997; 24: 201-210.

6. Gichangi,P.The syphilis screening control programme in Nairobi. Its impact on adverse obstetrical outcome and congenital syphilis. MMed. Thesis. UoN 1999.

7. Guay, L., Mmiro, F., Ndugwa, C.M., et al. The effect of HIV infection on the outcome of pregnancy in Ugandan women. J. Obstet. Gynae East Central Afr. 1993; 11: 32-37.

8. Omondi, L. B. Kumba. A comparative study of pregnancy outcome between HIV positive and HIV negative mothers at Kenyatta National Hospital. MMed Thesis. UoN 2001.

9. Jenny C. L., Msamanga, G. I., Mary Smith, C.F., et al. The association between maternal HIV -1 infection and pregnancy outcomes in, Dar es Salaam, Tanzania, Brit. J. Obstet. Gynaecol. 2001; 108: 1125-1133.
10. Nyongo, A., Gichangi, P., Temmermann, M., et al. HIV infection as a risk factor for chorioamnionitis in preterm birth. The VIII International Conference on AIDS, July 1992 Amsterdam. Abstract POB. 3469, P 3165.

11. Koonin, L. M., Ellerbrock, T. V., Atrash, H. K., et al. Pregnancy associated deaths due to AIDS in the United States. J. Amer. Med. Assoc. 1989; 261: 1306-1309.

12. Minkoff, H. L., Reget, R. H., Landesman, S., et al. Pneumocystis carinii pneumonia associated with AIDS in pregnancy. A report of 3 maternal deaths. Obstet. Gynecol. 1986; 67: 284-287.

13. Minkoff, H., Nanda, D., Menaz, R. and Firkrigs, R. Pregnancy resulting in infants with AIDS or AIDS related complex. Obstet. Gynecol. 1987; 69: 285-287.

14. Temmermann, M., Comba, E. N., Ndinya Achola, J., et al. Maternal HIV infection and pregnancy outcome. Obstet. Gynecol. 1994; 83: 495-501.

15. Chamiso, D. Pregnancy outcome in HIV-1 positive women in Gandhi Memorial Hospital Ethiopia. East Afr. Med. J. 1996; 73: 805-809.

16. Taha, T. E., Dallabeta, G. A., Canner, J. K., et al. The effect of Human Immunodeficiency Virus infection on birth weight and infant and child mortality in urban Malawi. Int. J. Epidemiol. 1995; 24: 1022-1029.

17. Marleen, T., Isaac, W., Peter, P. et al. A review of the impact of sexually transmitted diseases and HIV infection on pregnancy. outcome in Nairobi. J. Obstet. Gynecol. East Cent. Afr. 1991; 9: 55-57.

18. Ryder, R., Nga, W., Hassings, S., et al. Perinatal transmission of HIV-1 to infants of seropositive women in Zaire. N. Engl. J. Med. 1989; 320: 1637- 1642.

19. Merle, A., Sande and Volberding P. A. The medical management of AIDS. Fifth edition, W. B Saunders Company, 1997; 30: 459-468. 